\title{
Las habilidades blandas y su influencia en laconstrucción del aprendizaje significativo
}

\author{
MBA Lorena López López \\ UEBBR - Guayaquil \\ CTE - Guayaquil \\ lorenalopezlopez21@yahoo.com \\ Unidad Educativa Bilingüe Brisas del Río \\ Rectora \\ Comisión de Tránsito del Ecuador \\ Docente del Área de Administración \\ Msc Carla Lozano \\ UEBBR - Guayaquil \\ Docente/ CEO \\ White Star English Center
}

\section{RESUMEN}

Este artículo propone un análisis profundo sobre el aspecto socio emocional de losseres humanos en el cual las habilidades tanto blandas (soft skills) y duras (hard skills) propician un desarrollo integral del estudiante. El objetivo es determinar si el proceso de enseñanza-aprendizaje se realiza de forma idónea y si el dicente estálisto para poner en práctica el conocimiento transmitido a través de la demostración del progreso de las competencias al momento de analizar, sintentizar, comunicar y relacionarse en el grupo o con las demás personas.

Palabras clave: habilidades blandas; habilidades duras: empatía; valor; sintetizar. 


\title{
Soft skills and their influence on the construction of meaningful learning
}

\begin{abstract}
This article proposes a deep analysis of the social and emotional development of human beings in which soft and hard skills propitiate an integral development in students. The main objective is to determine if the teaching-learning process is accomplished in a suitable form and, if the pupil is ready to put in practice the knowledge taught by demonstrating the increasing of his/her abilities when he has to analyze, synthesize, communicate and interact with other people.
\end{abstract}

Keywords: soft skills; hard skills: empathy; value; synthesize

Artículo recibido: 05 octubre. 2021 Aceptado para publicación: 02 noviembre 2021 Correspondencia: lorenalopezlopez21@yahoo.com Conflictos de Interés: Ninguna que declarar 


\section{INTRODUCCIÓN}

Las habilidades blandas, cuántas veces nos hemos detenido a pensar en su importancia en el buen desarrollo tanto académico como personal de los seres humanos, y mucho más relevante si nos enfocamos en los estudiantes. ¿Los retos de la educación en todos los niveles nos han conllevado a estados de transición con la finalidad de adaptarnos de forma integral a las nuevas tendencias en el área de tecnología, estilos de vida, profesiones y áreas de trabajo, pero, estamos formando futuros seres humanos económicamente activos? O solamente un profesional más?.

Durante los últimos 18 meses la sociedad en general ha enfrentado grandes variaciones en el desenvolvimiento de sus actividades debido a la pandemiacovid-19, la misma que como todos estamos conscientes cada hecho en la vida nos deja transformaciones positivas y negativas las cuales han sido, son y continuarán siendo sobrellevadas y superadas con mucha creatividad, perseverancia y adaptación. Hemos leído y escuchado mucho sobre lashabilidades blandas en esta pandemia pero es necesario resaltar que éstas siempre han existido aunque su aplicación fue más limitada que ahora por ende su auge se lo ha visualizado durante este último tiempo.

La formación académica actual en el contexto y a nivel globalizado está caracterizada por tener como meta el desarrollo o potenciación de competencias tanto a nivel personal, académico y profesional teniendo como soporte el uso de las TIC y en la aplicación de metodologías o enfoques de aula que permitan un adecuado y oportuno proceso de enseñanza-aprendizaje.

Este artículo se centra en un entorno de diferentes niveles tales como: inicial, medio, superior, bachillerato y superior en variadas instituciones educativas, y que tiene como finalidad el estudio contemplativo del desenvolvimiento de competencias blandas en la enseñanza de los estudiantes para aportar positivamente en el alcance de los perfiles de salida requeridos para la continuidad de la educación superior tanto en el área cognitiva, destrezas y actitudes para lo cual deben ser solucionadores de problemas, creativos, críticos, innovadores, etc.

La importancia de este trabajo se focaliza en la indagación del desarrollo de habilidades blandas, individuales y sociales permitiendo estar listos parainsertarse de forma óptima en su carrera de tercer nivel y en el uso de metodologías en el aula virtual que promuevan y fomenten el aprendizaje. 


\section{DESARROLLO}

Debido a la relevancia que las habilidades blandas tienen en el desenvolvimiento de los seres humanos en alcanzar un desarrollo socioemocional idóneo, es relevante marcar diferencia entre las habilidades que debemos desarrollar y así poder enfrentar nuestras actividades de forma acertada. Sánchez, O. M., Amar, R. M., \& Triadú, J. X. (2018).

\section{¿QUÉ SON LAS HABILIDADES BLANDAS ?: según Maslow :}

Las habilidades blandas son el resultado de la combinación entre habilidades sociales, de comunicación, de personalidad, de cercanía a los demás, entre muchas, que forman a una persona capaz de relacionarse y comunicarse de manera efectiva con otros.

De acuerdo al concepto creado por el psicólogo estadounidense Abraham Maslow,creador de la Teoría de autorrealización, considera que a través de la aplicación delas habilidades blandas se lograrán vencer barreras permitiendo lograr una mejor yefectiva comunicación en un grupo y con los demás.

Las habilidades blandas o también llamadas no-cognitivas no son innatas del ser humano más bien son adquiridas y desarrolladas desde temprana edad, la infancia,con la ayuda de los padres desde casa y, posteriormente reforzadas en la institución educativa. El uso de dichas habilidades significa poner en práctica la aptitud, rasgos de personalidad y valores adquiridos que permitirán al ser humanotrabajar en equipo, adaptarse de forma flexible a situaciones cambiantes, serproactivos, autocríticos, empáticos, felices y, sobre todo exitosos: sin embargo, esnecesario enfatizar que no son trabajadas de forma directa sino de manera transversal, es decir se dan en todos los contextos de la vida. Ortega Goodspeed, T. (2016).

La aplicación de las habilidades blandas ha tomado mayor auge durante la pandemia covid 19 que hemos enfrentado puesto que la educación ha evolucionado según las necesidades emocionales detectadas por los docentes y el DECE dándole relevancia a la aplicación de las mismas y así alcanzar el enlace de valores transversales y el aprendizaje significativo. Huanaco Condori, K. J. (2019).

\section{Pero, ¿qué entendemos por "aprendizaje significativo"?}

\section{Según David Ausubel}

"El aprendizaje significativo es el conocimiento verdadero que nace cuando los nuevos contenidos tienen un significado a la luz de los conocimientos que ya se tienen". 
Por lo indicado en el concepto anterior creado por el psicólogo y pedagogodefensor de la psicología constructivista, se entiende que los nuevos aprendizajes se enlazan con los conocimientos previamente adquiridos. Cabe resaltar que el primordial punto en el aprendizaje significativo es que cada estudiante adquiere elnuevo conocimiento con un sentido único, es decir de forma clara a su propia interpretación lo cual facilita la retención del contenido puesto que ellos tendrán un rol participativo en la construcción del nuevo tema.

De acuerdo a lo manifestado por Lev Vygotsky (1987), el Desarrollo cognitivo está directamente relacionado con el contexto y baggage histórico, cultural y social. Puesto que, en su teoría, los procesos mentales superiores tienen su origen en la interacción social. De esta manera podemos establecer la importancia de las interacciones sociales para el desarrollo cognitivo de un individuo.

Es importante enfatizar que la aplicación idónea de las habilidades blandas nosolo permitirán alcanzar un aprendizaje significativo sino el desarrollo de competencias socioemocionales que darán paso a un desenvolvimiento adecuado en la sociedad, aspecto que todos los docentes anhelamos lograr en nuestros discentes Gómez-Gamero, M. E. (2019) En este punto es necesario reafirmar los conceptos de la neurociencia en la educación.

Neurociencia es el estudio de las emociones, y su objetivo es producir y regular emociones, pensamientos, conductas y funciones corporales básicas, Basados en ese concepto se crea el término NEUROEDUCACIÓN que es determinar cómo elsistema nervioso se activa en el momento de aprender. ESPINOZA, M., Antonio, M., GALLEGOS, B., \& del Pilar, D. (2020). Desde una perspectiva generalparece algo muy sencillo pero lamentablemente según nuestra cultura ylineamientos institucionales se les impide a los estudiantes exponer sus emociones durante el proceso de aprendizaje porque erróneamente se considera que los estudiantes solo deben expresarse en el momento que se les solicite puestoque se debe mantener orden en el aula, olvidando que al lograr interiorizar o no los objetivos cognitivos todas las sensaciones fluyen, el permitir exteriorizarlas serán la motivación para continuar en esa línea, es decir la inteligencia emocional se pone en evidencia. Siu, J. L. R. (2020).

La inteligencia emocional fue descrita por Salovey, P., \& Mayer, J. (1990) comola capacidad de los seres humanos de comprender y manejar las propias emociones y su 
influjo en los demás, procurando de esta manera un desarrollo tanto intelectual como emocional. Mencionando también, la anterioridad del desarrollo del cerebro emocional en relación al racional involucrando la posibilidad de que este sea una derivación del mismo y develando a su vez la importancia de su desarrollo y la relación entre pensamiento y sentimiento. Santos, C. E. O. (2017)

\section{METODOLOGÍA}

En un estudio realizado a un grupo de 43 docentes de la ciudad de Guayaquil cuyas edades fluctúan entre los 26 y los 59 años; por medio de una investigación de campo, entrevistas y encuestas, se pudo obtener la siguiente información sobre

el uso de las habilidades blandas en el desarrollo de sus clases tanto a nivel básico, bachillerato y superior.

Se utilizaron estrategias mixtas tanto cuantitativas como cualitativas durante el desarrollo de la encuesta a la que voluntariamente se sometieron los participantes de este estudio, teniendo las mismas preguntas personales generalizadas tales como su edad y profesión, así como también ocho preguntas de si y no o abiertas mediante las cuales se pudo profundizar tanto en la percepción de la importancia de las habilidades blandas en la educación y el conocimiento individual de las mismas.

\section{RESULTADOS}

Los resultados de esta investigación nos permiten tener claridad acerca de la comprensión e implementación de las habilidades blandas en la educación escolarizada. Las dos primeras preguntas se enfocaron en información personal como la edad y profesión, en cuanto a la carrera el $98 \%$ de los participantes se desempeñan en el campo de la docencia, siendo una minoría conformada por dos participantes quienes su área de trabajo se centra en la Psicología educativa e Ingeniería química respectivamente, pero reafirmando su validez puesto que sus labores se relacionan con el medio de la educación.

Es necesario mencionar que en la pregunta en la que inquiere si los participantes consideran que las emociones son influyentes en el desarrollo idóneo de los seres humanos todos los participantes respondieron sí, dándonos luces para inferir que la gran mayoría de docentes están conscientes de la importancia del desarrollo emocional de sus estudiantes puesto que están consciente que las habilidades blandas son el eje de un buen desenvolvimiento tanto personal como profesional del ser humano. En la pregunta “Considera usted que el mal manejo de las emociones son una de las principales causas 
que impiden alcanzar metas planteadas?" casi la totalidad de participantes manifestaron estar de acuerdo con la premisa, siendo esta una de las razones por las que es importante comprender,enseñar y conllevar a la aplicación del autocontrol de nuestras emociones.

\begin{tabular}{|l|c|c|}
\hline & Si & No \\
\cline { 2 - 3 } ¿Tiene conocimiento sobre las & 3 & 8 \\
habilidades delsiglo XXI, & 4 & \\
llamadas blandas? & & \\
\hline
\end{tabular}

Adicionalmente, podemos confirmar que 34 participantes de una totalidad de 43 manifestaron tener conocimiento de que son y cómo implementar las habilidades blandas o también conocidas como habilidades del siglo XXI cuyo uso ayuda al desarrollo de la inteligencia emocional, las cuales nos permiten comprender nuestra responsabilidad con nosotros mismos y con las personas a nuestro alrededor ayudando a mantener un mejor desenvolvimiento social, de esta manera se enriquece y potencia la labor docente a todo nivel, recordemos que desde las aulas se aporta a la formación holística de los futuros seres humanos profesionales. También pudimos visualizar que la gran mayoría de docentes participantes de este estudio, estaban de acuerdo en la relevancia de trabajar actividades que ayuden a desarrollar las habilidades blandas en niños desde la etapa escolarizada puesto que serán de gran utilidad en la formación integral delos individuos. Como lo podemos observar en la siguiente tabla, las razones por las cuales los docentes consideran que la implementación de las habilidades blandas y el desarrollo de las mismas para su futuro uso es beneficioso para los estudiantes, son variadas pero la mayoría absoluta se inclinó por afirmar que la razón principalera tener mejoras notables en el desarrollo de las emociones de los estudiantes, puntualizando también que es durante esta edad comprendida en la etapa escolarizada que se forma su personalidad y se desarrolla su madurez por ende ayudan en el proceso cognitivo e intelectual de los estudiantes facilitando el aprendizaje significativo.

Los docentes se inclinan a la aceptación e implementación de actividades que favorezcan el proceso de desarrollo del aprendizaje significativo en susestudiantes, puesto que es la manera en la que la información nueva o recientemente adquirida se relaciona con la parte cognitiva estructural del estudiante remarcando la importancia de un conocimiento previo 
de temas relacionados para comprender los nuevos. Los participantes mencionaron una amplia variedad de aplicaciones y herramientas tecnológicas que los asisten en el desarrollo de sus clases para favorecer dicha metodología.

\begin{tabular}{|c|c|}
\hline \multicolumn{2}{|c|}{$\begin{array}{c}\text { ¿Por qué considera que es relevante o no trabajar con las habilidades } \\
\text { blandas desde la etapaescolarizada? }\end{array}$} \\
\hline $\begin{array}{l}\text { Mejoras notables en el desarrollode las } \\
\text { emociones }\end{array}$ & 28 \\
\hline $\begin{array}{l}\text { Mejora las aptitudes y lacomunicación } \\
\text { en general }\end{array}$ & 4 \\
\hline $\begin{array}{l}\text { Ayudan en el proceso cognitivo yen el } \\
\text { desarrollo intelectual de losestudiantes }\end{array}$ & 9 \\
\hline
\end{tabular}

En referencia a la pregunta en la cual se consultaba sobre ¿Qué habilidades blandas conocen los encuestados ?, las respuestas fueron diversas, resaltando las más conocidas y aplicadas: comunicación, trabajo en equipo y liderazgo porque consideraron que eran los puntos neurálgicos para lograr un ambiente armónico tanto familiar como profesional. Es relevante delimitar que una buena comunicación en la cual exista una adecuada escucha se logrará una idóneacontestación permitiendo que esta fluya, y en este punto se debe reforzar mucho labuena escucha porque de esa manera se puede argumentar mejor y los resultados comunicativos serán exitosos. El trabajo en equipo el cual se realiza desde el período escolarizado hasta la etapa profesional pero en muchos de los casos se presentan inconvenientes durante el desarrollo del mismo debido a que todos los participantes no tienen interiorizados los valores de la responsabilidad y colaboración por ende no se trabaja de forma colaborativa sino individualizadamente, lo antes expuesto no posibilita la obtención de resultados satisfactorios y en algunas oportunidades concluye en una fricción muy marcada. La siguiente habilidad mencionada por los encuestados fue el liderazgo, en este punto se debe ser muy enfático en lo que el liderazgo es porque erróneamente las personas consideran que ser líder es ordenar, exigir e imponer y esto es el resultado de la no formación oportuna sobre los rasgos y el perfil que un caudillo debe poseer, y es justamente en este tema en donde se deben ver casi todas las habilidades blandas existentes conjugadas y puestas en evidencia. Es importante enfatizar que el liderazgo existe en todos los niveles por este motivo debe ser pulido hasta alcanzar que 
este sea el utilizado de forma competente.

Las habilidades blandas que también se mencionaron, pero en menor porcentaje son: empatía, resiliencia, adaptabilidad, colaboración y resolución de conflictos; el autocontrol de las mismas nos ayuda a alcanzar un mejor desenvolvimiento como ser humano, pero deben ser trabajadas desde la niñez.

\section{CONCLUSIONES}

Si bien es cierto las habilidades blandas han existido en todo momento de nuestras vidas pero no se les había otorgado la relevancia necesaria, y es a raíz de la pandemia covid 19 sumada a todos los contratiempos por el mal manejo de las emociones observados tanto desde la niñez hasta la adultez, los mismos que han encauzado a situaciones críticas y trágicas tales como alto índice de suicidios, frustraciones, estados depresivos, imposición de la manera de pensar, no adaptabilidad a los cambios; se determina la necesidad e importancia de trabajaren forma continua y firme con las habilidades blandas también llamadas del siglo XXI.

Es importante que cada docente incluya en su labor diaria el desarrollo de las habilidades blandas para que los niños y adolescentes aprendan a manejar sus emociones de forma coherente pues el autocontrol de su inteligencia emocionalles permitirá convivir en sociedad y alcanzar un desenvolvimiento profesional apto y acorde al momento.

La aplicación de las habilidades blandas durante la etapa escolarizada produce mejoras notables en el manejo saludable de las emociones y en la inteligencia emocional, la misma que es de vital importancia para el crecimiento integral delos seres humanos.

La inteligencia emocional nos faculta como seres funcionales y capaces de manejar diferentes situaciones que se pueden presentar a lo largo de la vida no solo académica y laboral si no también personal. 


\section{REFERENCIAS BIBLIOGRAFICAS}

Moreira, M. A., Caballero, M. C., \& Rodríguez, M. L. (1997). Aprendizaje significativo: un concepto subyacente. Actas del encuentro internacional sobre el aprendizaje significativo, 19(44), 1-16.

Salovey, P., \& Mayer, J. (1990). Inteligencia emocional. Imaginación, conocimiento y personalidad, 9(3), 185-211.

Sánchez, O. M., Amar, R. M., \& Triadú, J. X. (2018). Habilidades blandas:necesarias para la formación integral del estudiante universitario. Revista Científica Ecociencia, $5,1-18$.

Gómez-Gamero, M. E. (2019). Las habilidades blandas competencias para el nuevo milenio.Divulgare Boletín Científico de la Escuela Superior de Actopan, 6(11).

Siu, J. L. R. (2020). Las habilidades blandas como base del buen desempeño del docente universitario. INNOVA Research Journal, 5(2), 186-199.

Santos, C. E. O. (2017). Desarrollo de habilidades blandas desde edades tempranas". Journalof Selection and Assessment, 21(3), 251-263.

Huanaco Condori, K. J. (2019). Habilidades blandas y cuatro dominios en el marco del buendesempeño docente en la IE “Casa Blanca de Jesús” de SJL-2019.

ESPINOZA, M., Antonio, M., GAlleGOS, B., \& del Pilar, D. (2020). Habilidades blandas y su importancia de aplicación en el entorno laboral: perspectiva de alumnos de una universidad privada en Ecuador. Revista ESPACIOS. ISSN, 798, 1015.

Ortega Goodspeed, T. (2016). Desenredando la conversación sobre habilidadesblandas. Inter-American Dialogue.

VYGOTSKY, L. S. (1987). Pensamento e linguagem. $1^{\text {o }}$ ed. brasileira. São Paulo,Martins Fontes.

https://www.mejorespracticasrrhh.es/habilidades-blandas-como-desarrollarlas/ https://www.psicologia-online.com/habilidades-blandas-que-son-cuales-son-y-eje mplos5051.html

https://www.redalyc.org/jatsRepo/614/61458265007/html/index.html https://www.youtube.com/watch?v=ce87Wx6p3cU\&ab_channel=\%C2\%A1Noalf racasoescolar\%21 